\title{
Long-lived magnetoexcitons and two-dimensional magnetofermionic condensate in GaAs/AIGaAs heterostructure
}

 \\ Institute of Solid State Physics, Russian Academy of Sciences, \\ 142432 Chernogolovka, Russia \\ ฯ E-mail: gorbunov@issp.ac.ru
}

Excitation of long-lived triplet magnetoexcitons in a Hall insulator (filling factor $v=2$ ) with a high mobility of electrons, at low temperatures, $T<1 \mathrm{~K}$, enabled to discover a new collective state magnetofermionic condensate, that interacts coherently with an external electromagnetic field, exhibits superradiant properties and, owing to its low viscosity, spreads over the surface of the two-dimensional structure for macroscopically large distances.

\section{Acknowledgements}

We are grateful to S. Schmult (Max-Planck-Institut für Festkörperforschung, Stuttgart, Germany) for providing us with high-quality heterostructures and to S.M. Dickmann for useful discussions. The work was partially supported by the Russian Foundation for Basic Research. 